See discussions, stats, and author profiles for this publication at: https://www.researchgate.net/publication/343362924

\title{
Detonation-diffuse interface interactions: failure, re-initiation and propagation limits
}

Article in Proceedings of the Combustion Institute · August 2020

CITATIONS

0

3 authors:

Q. Josué Melguizo-Gavilanes

French National Centre for Scientific Research

64 PUBLICATIONS 205 CITATIONS

SEE PROFILE

(7) Brian McN. Maxwell

- Case Western Reserve University

57 PUBLICATIONS 180 CITATIONS

SEE PROFILE

Some of the authors of this publication are also working on these related projects:

Detonation waves View project

Project Flame acceleration and transition to detonation in unobstructed tubes/narrow channels. View project
READS

77

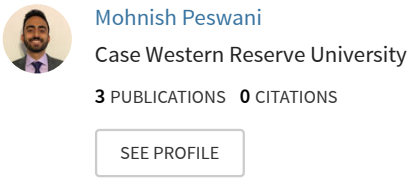




\title{
Detonation-diffuse interface interactions: failure, re-initiation and propagation limits
}

\author{
J. Melguizo-Gavilanes ${ }^{\mathrm{a}, *}$, M. Peswani ${ }^{\mathrm{b}}$, B.M. Maxwell ${ }^{\mathrm{b}}$ \\ ${ }^{a}$ Institut Pprime, UPR 3346 CNRS, ISAE-ENSMA, BP 40109, 86961 \\ Futuroscope-Chasseneuil Cedex, FRA \\ ${ }^{b}$ Department of Mechanical and Aerospace Engineering, Case Western Reserve \\ University, 10900 Euclid Avenue, Glennan 418, Cleveland, OH, 44106, USA
}

\begin{abstract}
Two-dimensional simulations of detonation interaction with gravity-driven diffuse interfaces, separating reactive mixtures and inert gas, were performed using the CLEM-LES methodology. The dynamics of the interactions were characterized and detonation failure and re-initiation mechanisms were described. Triple point transmission seems to be a characteristic feature of these configurations, and is responsible for both, quenching and re-ignition, within the diffuse interface. Results show that regions where the interface is thicker yield lower critical cell size gradients and vice versa. The average critical gradient found for the cases considered is 0.54 , higher than the value of 0.1 reported in literature. The extension of the limit appears to be a direct consequence of the cellular dynamics within the diffuse interface.
\end{abstract}

Keywords:

detonation-interface interaction, quenching limits, critical cell size gradient,

\footnotetext{
*Corresponding author:

Email address: josue.melguizo-gavilanes@cnrs.pprime.fr (J. Melguizo-Gavilanes)
} 
CLEM-LES, numerical simulation

\section{Introduction}

Studying detonation-interface interactions is relevant to industrial safety where upon accidental leaks/releases, layers of reactive and inert gases may form due to differences in molecular weight of the gases and/or insufficient mixing. The highly non-uniform conditions generated within the combustible cloud, as well as along its boundaries impose limitations on detonation propagation. Particularly, the presence of a diffusion layer at the edge of the cloud has been reported to increase the height of reactive layer required for successful propagation [1].

While detonation propagation limits in uniform mixtures has been studied extensively, non-uniform mixtures have received less attention [2]. This is mainly due to the experimental challenges associated with defining critical conditions for the latter. The characterization of the layer thickness at the interface where strong variations in equivalence ratio and dilution exist, as well as the specification of a unique cell width, $\lambda$, is not straightforward.

Gaseous detonations exhibit a characteristic cellular structure, which effectively describes the motion and collisions of transverse waves passing along the wave front forming triple points. Traditionally, detonations have been characterized as having either a regular or an irregular structure. Regular detonations have very structured patterns with cell widths that can be unambiguously determined. Irregular detonations on the other hand, exhibit stochastic-looking structures where various length scales are present [3-5]. $\lambda$ is directly correlated to the reactivity of the mixture where smaller $\lambda$ values 
are associated to faster reaction rates. As a result, $\lambda$ increases drastically for mixture compositions away from stoichiometry or with high dilution levels [6].

Detonation-interface interactions can be classified by comparing $\lambda$ with the interface thickness, $\delta$. A sharp interface occurs when $\lambda \gg \delta$ whereas a diffuse interface occurs when $\lambda \ll \delta$ or $\lambda \sim \delta$. Here, we are interested in numerically investigating the latter configuration in which the interface is generated by the opening of a sliding valve separating reactive from inert mixture. Small differences in molecular weights between the mixtures and buoyancy, result in a gravity current that forms a reactive layer whose reactivity gradually changes with height. The gradients in concentration lie perpendicular to the direction of detonation propagation, except at the head of the gravity current where gradients are parallel to the wave propagation.

Studies of detonation propagation into fuel concentration gradients aligned with the wave direction are numerous [7-11]; for gradients that lie perpendicular to it research is rather sparse [12-15]. Moreover, most, if not all, published numerical work to date is concerned with oversimplified scenarios in which only a few detonation cells are resolved, and has not been validated by nor compared with published larger-scale experiments.

In this manuscript we numerically examine the dynamics of detonationdiffuse interface interactions (following experiments in which industrially relevant scales are considered [14]), investigate failure/re-initiation mechanisms and report critical conditions for quenching within gravity-driven diffuse layers. 


\section{Computational methodology}

\subsection{Governing equations and models}

Simulations were conducted using the Compressible Linear Eddy Model for Large Eddy Simulation (CLEM-LES), a relatively new approach to modelling highly compressible and reactive flows. A detailed account of the methodology used, including the same mathematical formulation adopted here, can be found in [16]. Additional details regarding the methods performance and limitations are included in [17]. Here, for completeness, we provide a brief description. The flow is described by LES-filtered NavierStokes equations for a reactive calorically perfect gas. All state variables are normalized using the properties of the quiescent reactive mixture. The turbulent viscosity and dissipation are modeled according to

$$
\nu_{t}=\frac{1}{\pi}\left(\frac{2}{3 C_{\kappa}}\right)^{3 / 2} \sqrt{k^{s g s}} \bar{\Delta} ; \quad \epsilon=\pi\left(\frac{2 k^{s g s}}{3 C_{\kappa}}\right)^{3 / 2} / \bar{\Delta}
$$

where $\bar{\Delta}$ is the minimum grid spacing (equal to the LES filter size), $k^{s g s}$ is the subgrid kinetic energy, and $C_{\kappa}$ is the Kolmogorov number, a model parameter that requires calibration. The closure of the filtered reaction rate, $\overline{\dot{\omega}}$, is achieved using the CLEM subgrid modeling strategy [16]. The microscale mixing and chemical reaction are handled entirely on the sub-grid, through a supplementary simulation of a 1D sample of the flow field within each fully refined LES cell. For details on the system of equations solved on the sub-grid see [16].

A first order single-step Arrhenius reaction, $Y \rightarrow P$, is assumed, whose heat release is $Q$, and net consumption rate of reactants is given by $\dot{\omega}=$ $-\rho A Y \exp \left(-E_{a} / T\right) ; E_{a}$ and $A$ are the activation energy and pre-exponential 
factor, respectively. Only the reactants mass fraction $(Y)$ is transported, with values of zero corresponding to combustion products and/or inert gas.

\subsection{Model calibration}

For the single-step chemistry adopted, the model parameters, $Q / R T_{v N}=$ 23.64 and $E_{a} / R T_{v N}=5.47$, were tuned to reproduce the correct postshock ignition delay times, $\tau_{\text {ind }}$, for a fuel rich ethylene-oxygen mixture $\left(2.5 \mathrm{C}_{2} \mathrm{H}_{4}+3 \mathrm{O}_{2}\right)$ at an initial pressure and temperature of $p_{o}=11 \mathrm{kPa}$ and $T_{o}=300 \mathrm{~K}$. The ratio of specific heats $(\gamma=1.126)$ was determined at the detonation von-Neumann state $\left(T_{v N}=1975 \mathrm{~K}\right)$ for a shock travelling at the CJ-condition. Also, the pre-exponential factor $\left(A=1.33 \times 10^{8} \mathrm{~s}^{-1}\right)$, and diffusion coefficients $\left(\nu=2.54 \times 10^{-4} \mathrm{~m}^{2} / \mathrm{s}, P_{r}=0.77, P_{r, t}=1\right.$, and $\left.L e=1\right)$ were chosen such that the one-step model reproduced the correct half reaction length $\left(\Delta_{1 / 2}=0.1 \mathrm{~mm}\right)$, as well as the laminar flame speed at post-shock conditions, for a shock traveling at $50 \%$ of the theoretical CJ speed for the given quiescent mixture $\left(S_{L}=6.64 \mathrm{~m} / \mathrm{s}\right.$ at $\left.M_{D}=0.5 M_{C J}\right)$, see $[16,17]$ for details. This post-shock state was selected in order to have a measurable flame speed prior to auto-ignition of the gas. The reference values for $\tau_{\text {ind }}$, $\Delta_{1 / 2}$, and $S_{L}$ were determined with Cantera [18] using a chemical kinetic mechanism specifically designed for $\mathrm{C}_{2}$ and $\mathrm{C}_{3}$ chemistry [19]. Finally, $C_{\kappa}$ and $\bar{\Delta}$ (the LES filter size) required calibration to reproduce the average experimental cell size corresponding to the fuel mixture considered. The average cell structure obtained did not change beyond $\bar{\Delta}=\Delta_{1 / 2} / 8$, with the finest grid resolution of $\Delta_{1 / 2} / 128$ achieved on the CLEM subgrid, and a $C_{k}$ value of 1.5 . Figure 1 shows a numerically obtained soot foil for a detonation propagating into a uniform reactive mixture (no inert-gas interface present) 


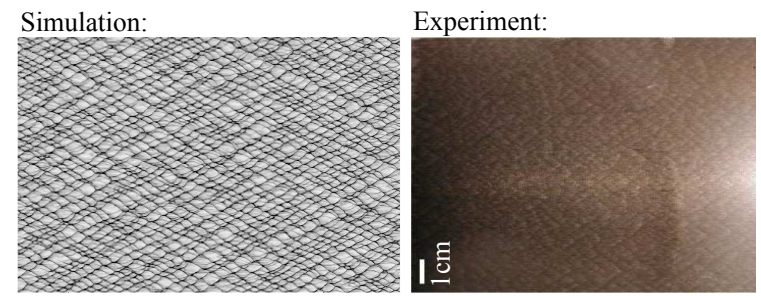

Figure 1: Numerical soot foil (left) compared to experiment [20] (right) in $2.5 \mathrm{C}_{2} \mathrm{H}_{4}+3 \mathrm{O}_{2}$. Cell sizes: $\lambda_{\text {sim }}=2.35 \mathrm{~mm} ; \lambda_{\exp }=2 \mathrm{~mm}$. Scale: $1 \mathrm{~cm}=100 \Delta_{1 / 2}$.

compared to scale with an experimental soot foil extracted from [20]. A mean cell size of $\lambda_{\text {sim }}=2.35 \mathrm{~mm}$ was found by postprocessing the numerical data using an autocorrelation procedure [21]; $\lambda_{\text {sim }}$ agrees well with the average reported value of $\lambda_{\exp }=2 \mathrm{~mm}$.

\subsection{Domain, initial and boundary conditions}

The total domain size of each simulation was 6100 to 8100 half reaction lengths $\left(\Delta_{1 / 2}\right)$ long $(x=0.61$ to $0.81 \mathrm{~m})$ by $1000 \Delta_{1 / 2}$ high $(y=100 \mathrm{~mm})$. This was nearly to scale with the experiments in $[14,20,22]$, which had a test section height of $y=150 \mathrm{~mm}$. The initial conditions were generated in a separate simulation in which an initially vertical interface separating reactive mixture $\left(2.5 \mathrm{C}_{2} \mathrm{H}_{4}+3 \mathrm{O}_{2}\right)$ and inert gas $\left(\mathrm{N}_{2}\right)$ was allowed to evolve naturally as a gravity current for $5 \mathrm{~s}$ to characterize its behavior. Accounting for molecular weight differences and buoyancy were necessary to mimic the sliding valve configuration used in [20]. To properly resolve the mixing/diffusion layer, a mixture averaged diffusion model based on computing binary diffusion coefficients from kinetic theory of gases was implemented [23]; this solver has been recently used in a hot surface ignition study performed by one of the authors [24]. The propagation speed of the head of the gravity current and 
the spatial horizontal/vertical growth of the interface throughout the domain were tracked during the simulation, and found to be in very good agreement with the values reported in [20] using a resolution of 10 points $/ \lambda_{\exp }$ (see Fig. 2). The spatial variation in mixing layer thickness, and their average values taken at 1 and $2.2 \mathrm{~s}$ after the opening of the sliding valve are also reported in Fig. 2. The gravity current from which the diffuse interface originates is computed resolving all the relevant scales using no-slip boundary conditions at the walls, and captures the Kelvin-Helmholtz instabilities that develop during its evolution as shown by the eventual roll-up of the interface visible in Fig. 3. Finally, the mass fraction of nitrogen, $Y_{\mathrm{N}_{2}}$, was used to map the gravity current simulation results to $Y$ via $Y=1-Y_{\mathrm{N}_{2}}$.

Detonation-diffuse interface interactions were investigated using the gravity current fields at two different times (see Fig.3). In each case, a corresponding ZND profile was initialized in the first $100 \Delta_{1 / 2}$ with $D=1.1 D_{C J}$ in order to overcome startup errors. The subsequent detonation front was permitted to propagate up to $x=2000 \Delta_{1 / 2}$ before encountering the interface, such that its quasi-steady velocity and cellular structure is established. Symmetric boundary conditions were specified on the top and bottom boundaries, while a no-slip wall boundary was specified at $x=-100 \Delta_{1 / 2}$. Note that for the detonation simulations, buoyancy effects were neglected since the characteristic propagation velocities of detonation waves $\left(u_{\operatorname{det}} \sim 10^{3} \mathrm{~m} / \mathrm{s}\right)$ are four orders of magnitude higher than those of gravity currents at the scale considered here $\left(u_{\text {gc }} \sim 10^{-1} \mathrm{~m} / \mathrm{s}\right)$. Simulations were run using 250 processors per case and took approximately 250,000 CPU hours in total. 

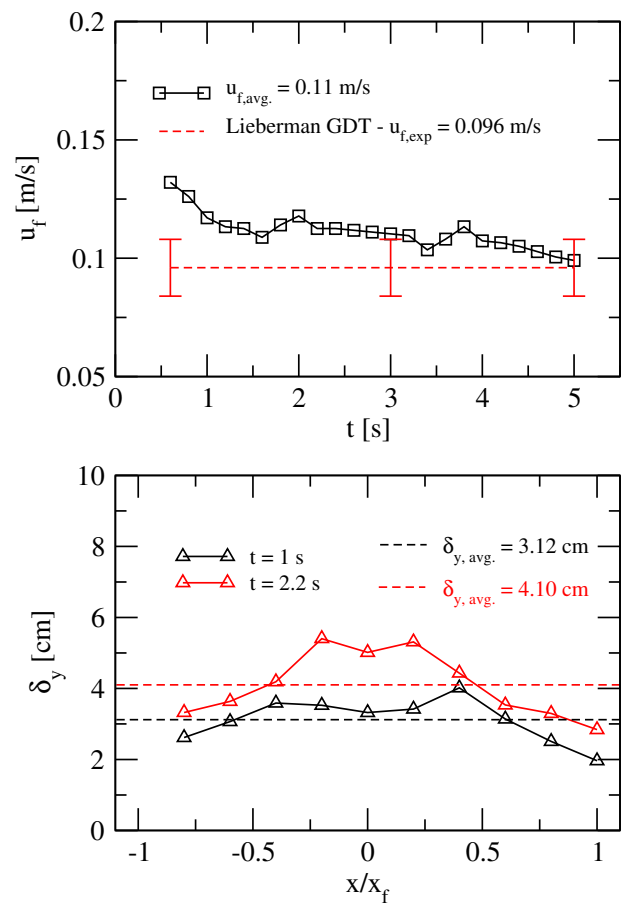

Figure 2: Characterization of gravity current for determining initial conditions - Top: propagation speed of head of gravity current, $\mathrm{u}_{\mathrm{f}}$, vs. time. Bottom: interface vertical thickness, $\delta_{\mathrm{y}}$, vs. distance normalized by gravity current's head position, $\mathrm{x}_{\mathrm{f}}$, at the time considered.

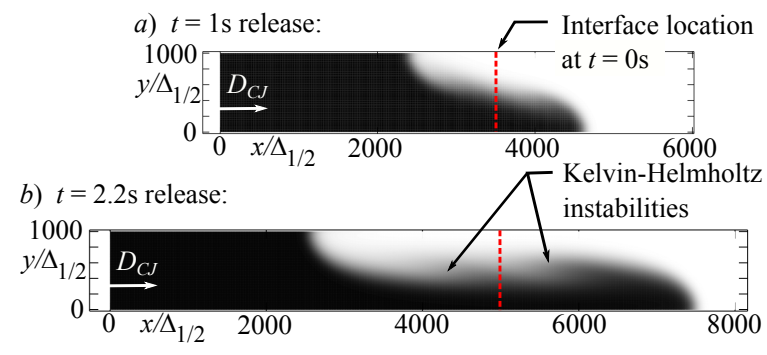

Figure 3: Initial fields for detonation-diffuse interface interactions. Dark regions represent reactive fuel-oxidizer mixture $(Y=1)$; white regions are inert gas and/or detonation products $(Y=0)$. 


\section{Results and Discussion}

\subsection{Flow structure: experiment vs. simulation}

Figure 4 shows a qualitative comparison of the flow structure numerically predicted with that visualized experimentally [20]. The fields shown are those for a detonation interacting with the gravity current in Fig. 3b. The main features of this wave complex are: (i) a curved detonation front; (ii) a transmitted oblique shock wave (TS); and (iii) a trailing turbulent mixing zone (TMZ). Note that due to confinement a Mach reflection, a transverse and reflected shock wave are also visible. The simulations offer additional insight

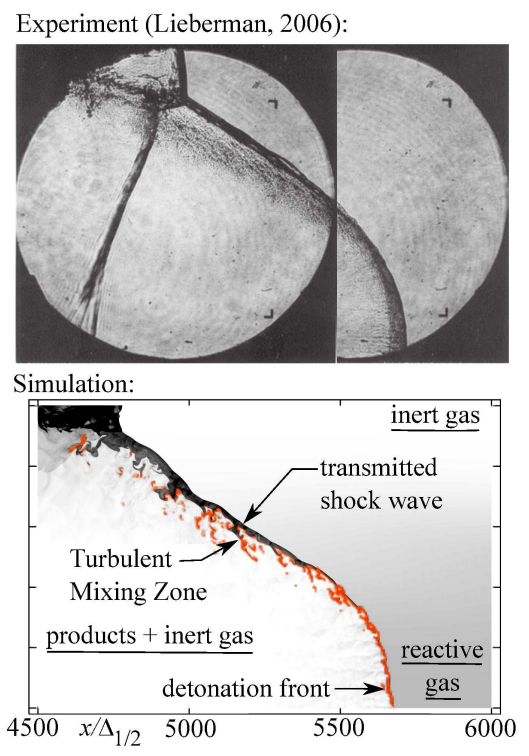

Figure 4: Qualitative comparison of experimental [20] and numerical flow structure. Experimental viewing window: $150 \mathrm{~mm}$ diameter; simulation domain height: $100 \mathrm{~mm}$.

by distinctly showing regions within the TMZ where chemical reactions are active (red areas) caused by the TS processing a layer of decreasing reactivity. This is in contrast with the flow structure observed in detonation-sharp 
interface interactions where no chemical activity is present in the TMZ because the TS processes a layer of inert gas only [20]. In the latter case, the TMZ merely serves as an interface that separates burned from shocked inert gas. The flow structure for detonation interaction with the gravity current at $t=1 \mathrm{~s}$ is essentially the same except that the TMZ and TS angles are slightly lower when measured using the standard convention (i.e. counter clockwise from positive $x$-axis).

\subsection{Overall dynamics}

Numerical soot foils were obtained by tracking the vorticity magnitude maxima in the computational domain, per cell at each time step. They provide a time history of the triple point trajectories during detonation transmission through the diffuse interface, and allow to determine regions where local quenching and re-initiation events occur. The full detonation-diffuse interface evolution for detonation interaction with the gravity current at $t=1 \mathrm{~s}$ and $t=2.2 \mathrm{~s}$ after the opening of the sliding valve is shown in Fig. $5 \mathrm{a}$ and b, respectively. In both soot foils, triple-point transmission is observed with an associated increase in cell size. The main physical mechanisms that contribute to the cell size increase are unsteady expansion waves [25] coming from the diffuse interface, and the decreasing reactivity of the mixture along the diffuse interface $(Y<1)$ which physically arises from changes in equivalence ratio and dilution that take place during the development of the gravity current itself. The end result of these two processes is a reaction rate reduction due to expansion induced cooling for the former, and variations in total energy content for the latter. Bear in mind that the results presented here with the single-step model used only mimic dilution variations. 


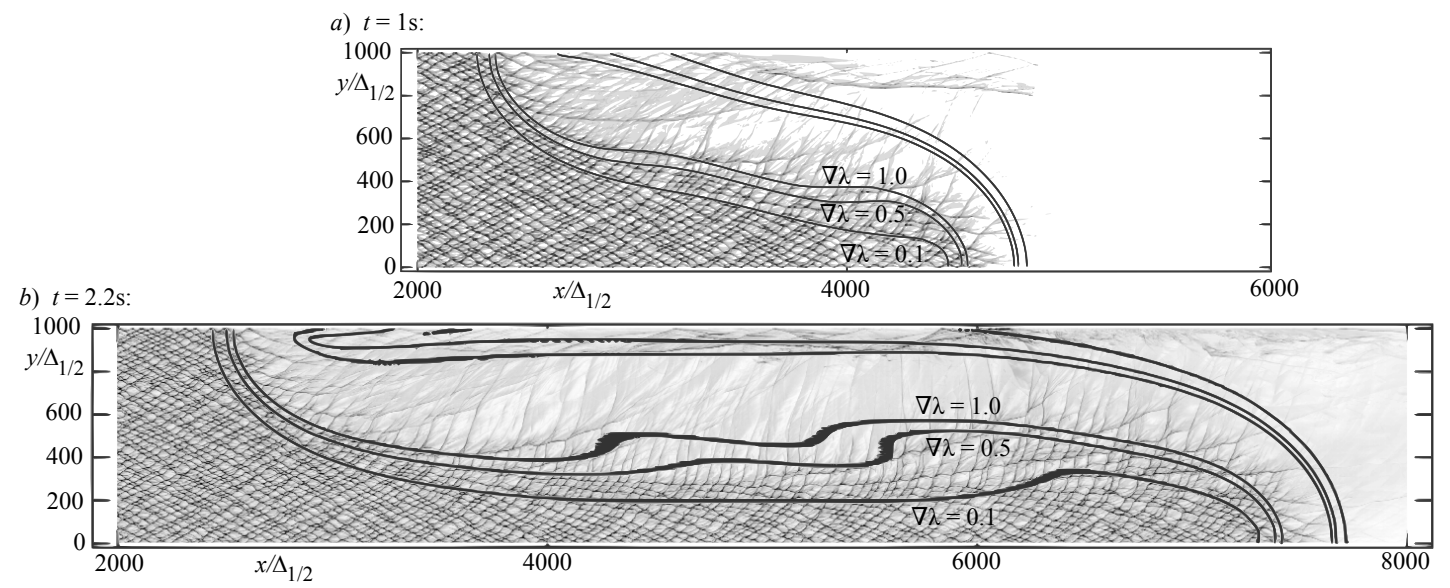

Figure 5: Numerical soot foils showing detonation-diffuse interface interaction with gravity current at two different times after opening of sliding valve $-t=1 \mathrm{~s}$ (top); $t=2.2 \mathrm{~s}$ (bottom). Solid lines are contours of cell size gradient, $\nabla \lambda$, at $0.1,0.5$ and 1.0.

The soot foil in Fig. 5b shows the most interesting dynamics with three distinct regions: (i) presence of runaway triple points for $3500 \leq x / \Delta_{1 / 2} \leq$ 4500 that subsequently decay as they approach the end of the diffuse layer $\left(y / \Delta_{1 / 2} \sim 500\right)$; (ii) undisturbed propagation for $4500 \leq x / \Delta_{1 / 2} \leq 5000$ analogous to that seen for detonation propagation under yielding confinement [25] with no visible cells along the interface thickness (likely quenching), immediately followed by additional triple point transmission at $x / \Delta_{1 / 2} \sim 5000$, and 5500; (iii) re-initiation of the detonation, within the diffuse layer, upon collision of the previously transmitted triple points with new ones at $x / \Delta_{1 / 2} \sim$ 6000 . These observations, in line with previous work [9, 26], seem to suggest that the diffuse interface thickness and its vertical concentration gradient govern the quenching, successful triple point transmission and re-initiation of the front within the interface. 


\subsection{Critical conditions}

Detonation propagation limits for uniform mixtures are typically given as a function of a characteristic length, $\ell$, dictated by the configuration considered, scaled by the detonation cell size, $\lambda$. For confined tubes and channels the critical conditions are $\ell \geq \lambda / \pi$ and $\ell \geq \lambda$, respectively. For detonation transmission from tubes/channels to open-space, $\ell$ is $13 \lambda$ and $11 \lambda$ for most hydrocarbons, and reduces to $\ell=3 \lambda$ for high aspect ratio channels; the latter critical value was also found for detonation propagation in stoichiometric hydrogen-oxygen mixtures confined by an inert layer [27], for hydrocarbons, this value increases to $\ell \sim 5-10 \lambda$ [28]. For non-uniform mixtures such as the gravity currents considered here, determining critical conditions poses additional challenges because the mixture reactivity changes along the boundaries of the current making it difficult to define a unique value of $\lambda$. Previous work has shown that strong gradients in reactivity are conducive to quenching, and that these reactivity gradients can be directly mapped to detonation cell size gradients [9, 26]. While the critical cell size gradient has been documented to depend on the mixture thermodynamic properties, and

found to decrease with increasing $E_{a} / R T_{v N}$, as well as dimensionality [26], the mean value reported in literature, generally accepted in the safety community, is $\nabla \lambda \sim 0.1$ [9]. For the current investigation it is not possible to compare the critical cell size gradient directly to the experiments in $[14,20]$ since such information is not available. Instead, we compare the quenching criteria found from our simulations with those reported in [9], even though the experimental setup used is different.

To test the validity of this metric for our case, additional simulations 
were run for planar detonations using uniform conditions, such as that conducted in Fig. 1, to map the expected increase in cell size, $\lambda$, as a function of initial reactant mass fraction, $Y$ (i.e. reactive mixtures diluted with different amounts of inert gas). The results are shown in Fig. 6. The cell size was measured both manually and automatically (using the autocorrelation procedure described in [21]), and subsequently fitted using an exponential function. The mathematical form of the fit was chosen based on the reported experimental dependence of cell size as a function of nitrogen dilution for ethylene-oxygen mixtures [6]. Finally, the field of cell size gradient can be computed from the gravity current detonation simulations through the expression $\nabla \lambda=(d \lambda / d Y) \cdot \nabla Y$.

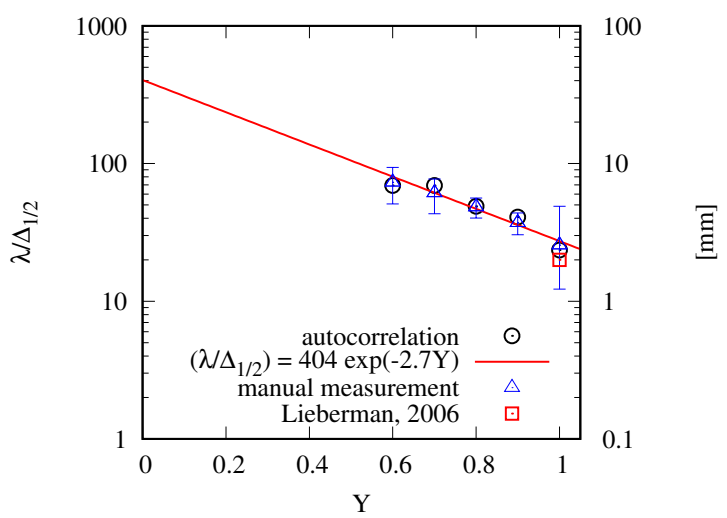

Figure 6: Detonation cell size vs. reactants mass fraction $(Y)$ obtained by conducting planar detonation wave simulations in uniform mixtures.

The results presented in Fig. 5, were sampled at 250 intervals along the $x / \Delta_{1 / 2}$ axis. Representative plots of mass fraction and vorticity magnitude maxima, together with the vertical gradient of cell size and mass fraction are included in Fig. 7. The vertical dashed-dotted line is a visual aid indicating 
the end of the cellular structure, signaled by a drop in fluctuations in the $|\omega|_{\max }$ profile. The intersection of this line with the $\nabla \lambda$ curve permits to uniquely determine the cell size gradient at which quenching occurs. In most cases, it corresponds to the location where the first decoupling of the shock and reaction zone is observed in the numerical sootfoil records. Except, when runaway triple points occur, as discussed in the next section. The samples
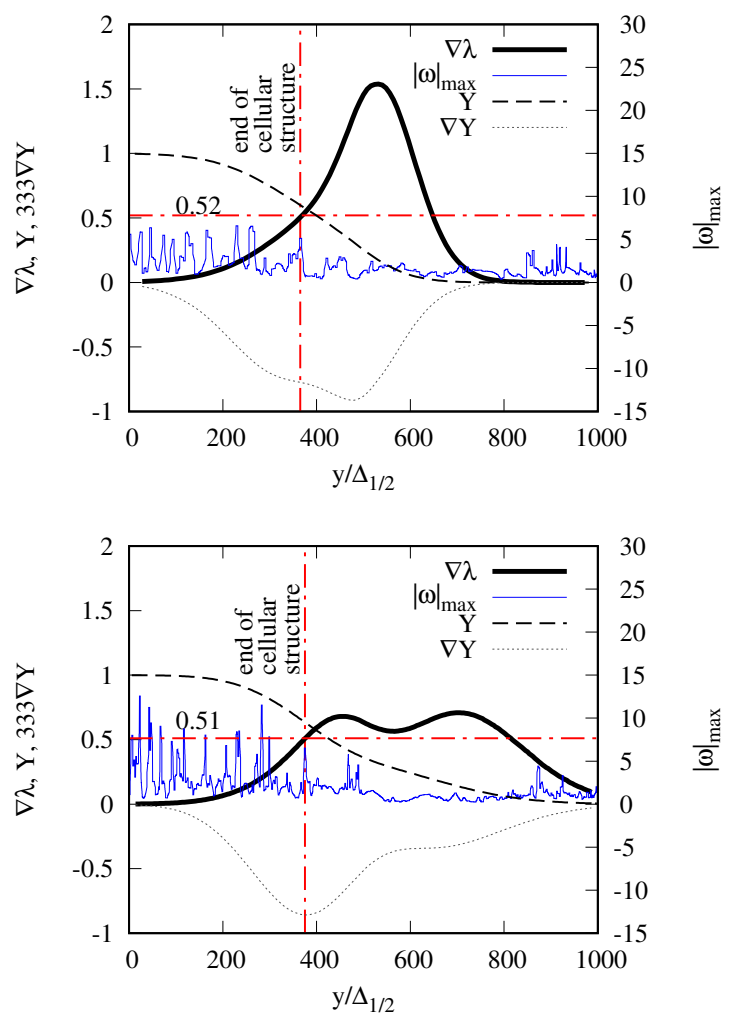

Figure 7: Vorticity, mass fraction, vertical gradient of cell size and mass fraction profiles at $x / \Delta_{1 / 2}=4000$ for detonation-diffuse interface interaction with gravity current after $1 \mathrm{~s}$ (top) and $2.2 \mathrm{~s}$ (bottom) of sliding valve opening.

taken at $x / \Delta_{1 / 2}=4000$ for both detonation-diffuse interface interactions 
yield critical cell size gradients for quenching, $(\nabla \lambda)_{\text {crit }}$, of 0.52 and 0.51 , respectively. Fig. 8 summarizes all our results. The average $(\nabla \lambda)_{\text {crit }}$ are 0.63 and 0.44 significantly higher than the $\nabla \lambda \sim 0.1$ criteria previously proposed, making it a rather conservative estimate for this type of scenario. Contours of $\nabla \lambda=0.1,0.5$, and 1.0 are shown in Fig. 5 for reference. Note that most of the cellular activity, including runaway triple points are enclosed within $\nabla \lambda \leq 0.5$ region for both cases. Finally, the critical cell size gradient,

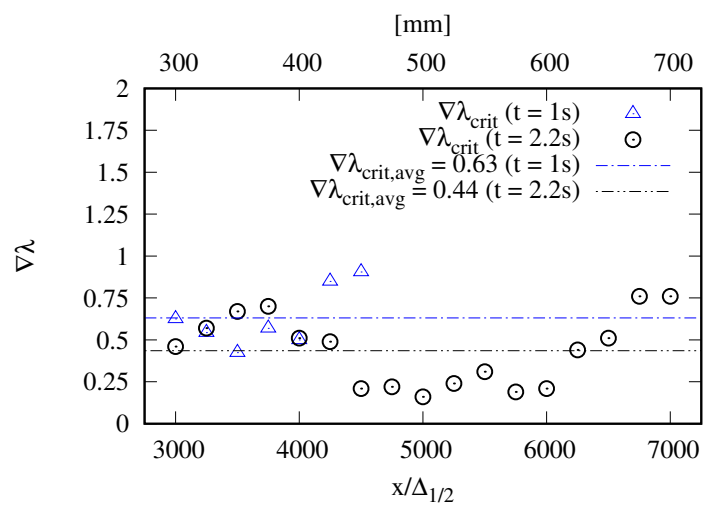

Figure 8: Critical cell size gradient, $\nabla \lambda_{\text {crit }}$, vs. normalized distance, $x / \Delta_{1 / 2}$, for detonation-diffuse interface interaction with gravity current after $1 \mathrm{~s}(\triangle)$ and $2.2 \mathrm{~s}(\odot)$ of sliding valve opening.

$(\nabla \lambda)_{\text {crit }}$, is plotted against the corresponding vertical interface thickness, $\delta_{y}$, in Fig. 9; $\delta_{y}$ was determined at each $x$-location by measuring the vertical distance between $0.1 \leq Y \leq 0.9$. These two quantities seem to be inversely correlated: thicker reactive layers require smaller cell size gradients, and vice versa. Surprisingly, $\nabla Y$ alone was not found to correlate with detonation quenching through the diffuse layer. 


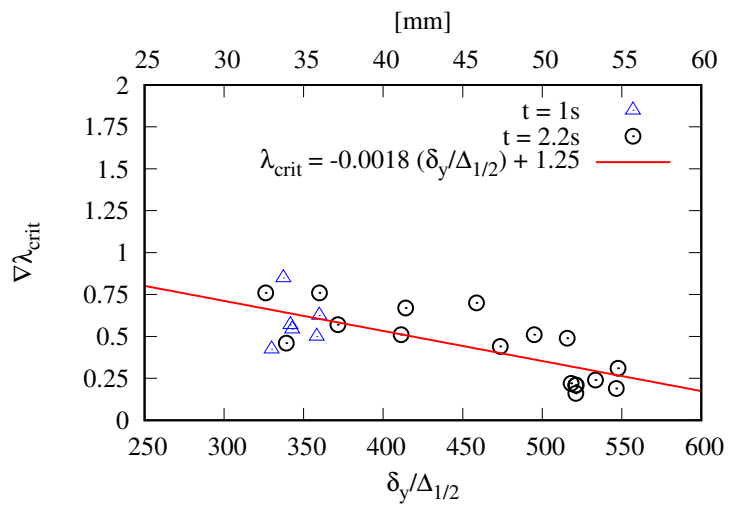

Figure 9: Critical cell size gradient, $(\nabla \lambda)_{\text {crit }}$ vs. diffusion layer thickness, $\delta_{y} / \Delta_{1 / 2}$.

\subsection{Quenching/re-initiation mechanisms}

The dynamics described in subsection 3.2 warrants a deeper investigation into the regions where quenching and re-initiation events were observed within the diffuse interface. In Fig. 10 a close up to partial quenching is

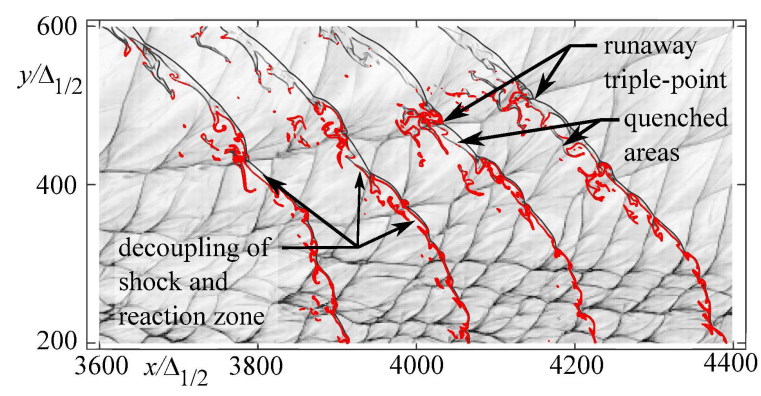

Figure 10: Instantaneous fields of density gradient and reaction rate overlaid on soot foil. Close up to partial quenching, $x / \Delta_{1 / 2} \in[3600-4400] ; y / \Delta_{1 / 2} \in[200-600]$, for detonation-diffuse interface interaction with gravity current after $t=2.2 \mathrm{~s}$ of sliding valve opening.

shown. Instantaneous fields of density gradient and reaction rate overlaid on the soot foil clearly displays triple points detaching from the main detonation 
front, running away through the diffuse interface. As they propagate into a layer of decreasing reactivity, the leading shock and reaction zone progressively decouple until chemical heat release is no longer capable of sustaining the front. The triple points and leading shock weaken as a result. In the absence of triple point reflections from the interface or top wall, the detonation ultimately quenches within the layer. Triple point transmission seems to be

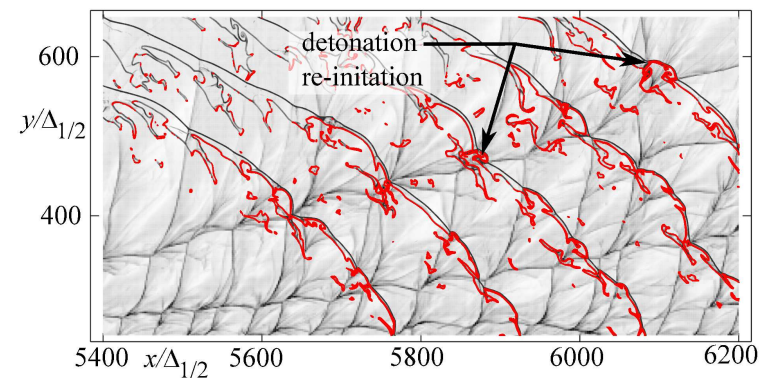

Figure 11: Instantaneous fields of density gradient and reaction rate overlaid on soot foils. Close up to re-initiation, $x / \Delta_{1 / 2} \in[5400-6200] ; y / \Delta_{1 / 2} \in[250-650]$, for detonationdiffuse interface interaction with gravity current after $t=2.2 \mathrm{~s}$ of sliding valve opening.

a characteristic feature of detonation propagation through diffuse layers, in contrast to what is typically observed for detonation interactions with sharp interfaces (i.e. triple point reflection) [25]. Shock transmission/reflection phenomena is typically linked to variations in acoustic impedance ratios across interfaces. Based on our model assumptions, however, this ratio is unity. The triple point transmission observed is therefore due to having a layer of finite thickness and decreasing reactivity. The gravity currents considered here exhibit a variable layer thickness (see Fig. 2). The longer the time allowed for their development, the higher the likelihood of encountering thicker regions where transmitted triple points, at different instances during 
the detonation-interface interaction, can coalesce and generate local thermodynamic conditions conducive to detonation re-initiation (i.e. high $p$ and $T$ ). This can occur even if the reactivity within the layer is low. This feature is displayed in Fig. 11.

\subsection{Applicability of the reported $(\nabla \lambda)_{\text {crit }}$ values}

In agreement with the previous experimental and numerical work cited throughout the manuscript, and the references therein, thicker interfaces favor detonation transmission. The reported $(\nabla \lambda)_{\text {crit }}$ values are not to be taken as hard limits as they vary as a function of $\delta_{y}$ (see Fig. 9). Moreover, for mildly irregular mixtures, there is significant uncertainty in determining a fixed cell size, even in the absence of reactivity gradients; these variations also need being accounted for. The generally accepted critical cell size gradient of $(\nabla \lambda)_{\text {crit }} \sim 0.1$ gives a good estimate of the regions below which undisturbed detonation propagation takes place (see Fig 5). However, the importance of the cellular dynamics within the layer, which had been suggested but not explored in $[9,26]$, seems to be responsible for the higher

critical values found (see section 3.4). Finally, it is important to emphasize that different descriptions of the chemistry in the numerical modeling may yield different critical conditions, as was discussed at length in [29].

\section{Conclusion}

Two-dimensional simulations of detonation interaction with diffuse interfaces were performed using the CLEM-LES methodology. The diffuse interfaces were generated in a separate simulation that captured the gravity 
currents that develop after the opening of a sliding valve separating reactive from inert mixture. The flow structure obtained was compared with experimental results performed at industrially relevant scales, and found to be in very good agreement. This is seldom done in the community and was possible because of the numerical framework used. The dynamics of the interaction were characterized and detonation failure and re-initiation mechanisms described; triple point transmission seems to be a characteristic feature of these configurations in contrast to what is typically observed for interaction with sharp interfaces. Critical cell size gradients for detonation failure were computed along the interface, and compared with a metric available in literature i.e. $(\nabla \lambda)_{\text {crit }} \sim 0.1$. Our results indicate that regions where the interface is thicker yield lower $(\nabla \lambda)_{\text {crit }}$ and vice versa. The average $(\nabla \lambda)_{\text {crit }}$ of both cases considered is 0.54 . Future work will focus on quantifying, in terms of interface thickness/reactivity gradient, the limit for triple point transmission vs. reflection.

\section{Acknowledgements}

This research was enabled in part by high performance computing resources provided the Core Facility for Advanced Research Computing at Case Western Reserve University.

\section{References}

[1] A. Vasil'ev, D. Zak, Detonation of gas jets, Combustion, Explosion, and Shock Waves 22 (1986) 463-468.

[2] J. H. Lee, The detonation phenomenon, 2008. 
[3] R. Strehlow, Gas phase detonations: Recent developments, Combust. Flame 12 (1968) 81-101.

[4] J. M. Austin, The role of instability in gaseous detonation, Ph.D. thesis, California Institute of Technology, Pasadena, CA, 2003.

[5] F. Pintgen, C. A. Eckett, J. M. Austin, J. E. Shepherd, Direct observations of reaction zone structure in propagating detonations, Combust. Flame 133 (2003) 211-229.

[6] M. Kaneshige, J. Shepherd, Detonation database, GALCIT Report FM97-8, California Institute of Technology: Aeronautics and Mechanical Engineering, 1997.

[7] R. A. Strehlow, A. A. Adamczyk, R. J. Stiles, Transient studies of detonation waves, Astronautica Acta 17 (1972) 509-527.

[8] G. Thomas, P. Sutton, D. Edwards, The behavior of detonation waves at concentration gradients, Combustion and Flame 84 (1991) 312 - 322.

[9] M. Kuznetsov, V. Alekseev, S. Dorofeev, I. Matsukov, J. Boccio, Detonation propagation, decay, and reinitiation in nonuniform gaseous mixtures, in: Symposium (International) on Combustion, volume 27, Elsevier, pp. 2241-2247.

[10] E. S. Oran, D. A. Jones, M. Sichel, Numerical simulations of detonation transmission, Proc. R. Soc. Lond. A 436 (1992) 267-297.

[11] N. A. Tonello, M. Sichel, C. W. Kauffman, Mechanisms of detonation transmission in layered $\mathrm{H}_{2}-\mathrm{O}_{2}$ mixtures, Shock Waves 5 (1995) 225-238. 
[12] D. A. Kessler, V. N. Gamezo, E. S. Oran, Gas-phase detonation propagation in mixture composition gradients, Phil. Trans. R. Soc. A 370 (2012) 567-596.

[13] K. Ishii, M. Kojima, Behavior of detonation propagation in mixtures with concentration gradients, Shock Waves 17 (2007) 95-102.

[14] D. H. Lieberman, J. E. Shepherd, Detonation interaction with a diffuse interface and subsequent chemical reaction, Shock Waves 16 (2007) 421-429.

[15] L. R. Boeck, F. M. Berger, J. Hasslberger, T. Sattelmayer, Detonation propagation in hydrogen-air mixtures with transverse concentration gradients, Shock Waves 26 (2016) 181-192.

[16] B. M. Maxwell, R. R. Bhattacharjee, S. S. M. Lau-Chapdelaine, S. A. E. G. Falle, G. J. Sharpe, M. I. Radulescu, Influence of turbulent fluctuations on detonation propagation, J. Fluid Mech. 818 (2017) 646-696.

[17] B. Maxwell, A. Pekalski, M. Radulescu, Modelling of the transition of a turbulent shock-flame complex to detonation using the linear eddy model, Combust. Flame 192 (2018) 340-357.

[18] D. G. Goodwin, H. K. Moffat, R. L. Speth, Cantera: An object-oriented software toolkit for chemical kinetics, thermodynamics, and transport processes, http://www . cantera.org, 2016. Version 2.2.1.

[19] H. Wang, A. Laskin, Z. M. Djurisic, C. K. Law, S. G. Davis, D. L. Zhu, A comprehensive mechanism of $\mathrm{C} 2 \mathrm{Hx}$ and $\mathrm{C} 3 \mathrm{Hx}$ fuel combustion, in: 
Chemical and Physical Processes of Combustion, the 1999 Fall Technical Meeting of the Eastern States Section of the Combustion Institute, Raleigh, NC, pp. 129-132.

[20] D. Lieberman, Detonation interaction with sharp and diffuse interfaces, Ph.D. thesis, California Institute of Technology, Pasadena, CA, 2006.

[21] G. J. Sharpe, M. I. Radulescu, Statistical analysis of cellular detonation dynamics from numerical simulations: one-step chemistry, Combustion Theory and Modelling 15 (2011) 691-723.

[22] D. H. Lieberman, J. E. Shepherd, Detonation interaction with an interface, Phys. Fluids 19 (2007) 096101.

[23] R. J. Kee, M. E. Coltrin, P. Glarborg, Chemically reacting flow: theory and practice, John Wiley \& Sons, 2005.

[24] S. A. Coronel, J. Melguizo-Gavilanes, R. Mével, J. E. Shepherd, Experimental and numerical study on moving hot particle ignition, Combustion and Flame 192 (2018) 495-506.

[25] M. Reynaud, F. Virot, A. Chinnayya, A computational study of the interaction of gaseous detonations with a compressible layer, Physics of Fluids 29 (2017) 056101.

[26] S. Kryuchkov, S. Dorofeev, A. Efimenko, Critical conditions for detonation propagation through mixture with decreasing reaction rate, in: Symposium (International) on Combustion, volume 26, Elsevier, pp. 2965-2972. 
[27] W. Rudy, M. Kuznetsov, R. Porowski, A. Teodorczyk, J. Grune, K. Sempert, Critical conditions of hydrogen-air detonation in partially confined geometry, Proc. Combust. Inst. 34 (2013) 1965-1972.

[28] A. Borisov, S. Khomic, V. Mikhalkin, Detonation of unconfined and semiconfined charges of gaseous mixtures, Prog. Astronaut. Aeronaut 133 (1991) 118-132.

[29] S. Taileb, J. Melguizo-Gavilanes, A. Chinnayya, Influence of the chemical modeling on the quenching limits of gaseous detonation waves confined by an inert layer, Combustion and Flame (2020). 